\title{
Physiological Homeostasis Alteration and Cellular Structure Damage of Chlorella Vulgaris Exposed to Silver Nanoparticles with Various Microstructure Morphologies
}

\author{
Ningcan Deng \\ Northeastern University \\ Haibo Li ( $\nabla$ lihaibo@mail.neu.edu.cn ) \\ Northeastern University \\ Yinghua Li \\ Northeastern University \\ Fan Mo \\ Northeastern University \\ Mingshuai Wang \\ Northeastern University \\ Zhe Li \\ Northeastern University \\ Xi Chen \\ Northeastern University \\ Jianing Xu \\ Northeastern University \\ Rui Chai \\ Northeastern University \\ Hongxuan Wang \\ Northeastern University
}

\section{Research Article}

Keywords: silver nanoparticles, Chlorella vulgaris, structure-effect relationship, microstructure morphology

Posted Date: June 3rd, 2021

DOl: https://doi.org/10.21203/rs.3.rs-508869/v1

License: (9) This work is licensed under a Creative Commons Attribution 4.0 International License. Read Full License 
Page $2 / 12$ 


\section{Abstract}

The toxicity of silver nanoparticles (AgNPs) with single morphology exposed to aquatic organisms had been well revealed in the past decade, but few studies have been carried out to evaluate the toxicity differences between AgNPs with various microstructure morphologies, especially to algae. In this work, Chlorella vulgaris was used as the tested organism to illustrate the differences of toxic effects between silver nanospheres (AgNSs), silver nanocubes (AgNCs) and silver nanoplates (AgPLs) with concentration of $0.5,1.0,2.0,5.0{\mathrm{mg} \otimes \mathrm{L}^{-1}}^{-1}$, based on the algae's growth (72h), chlorophyll-a content, antioxidant enzyme activity, lipid peroxidation and cell apoptosis (48h). The results showed that the toxicity level exposed to Chlorella vulgaris was in the order of AgPLs> AgNCs> AgNSs. The difference shown indicated that the potential toxicity of AgNPs is primarily depended on their microstructure morphologies. This current study initially revealed the structure-effects of AgNPs on Chlorella vulgaris, provided a scientific basis for aquatic environmental risk assessment.

\section{Introduction}

Silver nanoparticles (AgNPs) produced, transported and applicated by humans are ultimately released into the environment and are thus potentially toxic to environmental organisms (Dale et al., 2015). Besides, in natural environment, $\mathrm{Ag}^{+}$can be reduced into nano silver by dehydrogenase or reducing sugar in microorganisms or plants (Mo et al., 2020). AgNPs is highly chemically active and can easily interact with environmental medium (e.g., physical, chemical and biological reaction), which leads to the migration and transformation of AgNPs, eventually affecting their toxic effects (Wang et al. 2020). Although much work was gone into focusing on the biological effects of AgNPs, which systematically revealed action mechanisms and dose-effect relationship of AgNPs with single morphology (e.g., spherical, rodlike, and hexagonal) to various organisms, the shape-dependent toxicity was less explored (Ji et al. 2010; Oukarroum et al. 2012). Noticeably, there is an internal relationship between the structure of pollution and their toxic effect, that is, the structure-activity relationship. Different morphologies of nanoparticles possess different crystal planes and quantum structures, leading to the unique physical and chemical properties (Shen et al. 2015). Several studies have evaluated the ecotoxicity of AgNPs with various morphologies to bacterial, algae and fish. Mayer et al. demonstrated that AgNPs morphologies had no significant effect on the cytotoxicity (2016), while Babak et al. compared the toxicities of silver nanoplates (AgPLs) and silver nanospheres (AgNSs) and found that AgNSs were highly toxic to Staphylococcus aureus and Escherichia coli (E. coli), yet less toxic than AgPLs (2012). In contrast, some studies reported that the toxicities of AgPLs and AgNSs in P. aeruginosa and E. coli were opposite (Muhammad et al. 2016). Therefore, there is a dispute related to shape-dependent toxicity of AgNPs. Thus, it is of great importance to explore the toxic differences of AgNPs with various morphologies on organisms in ecosystem, providing direct evidence for the risk assessment of AgNPs with different structure.

As a main primary producer in aquatic systems, algae play a crucial role in the environmental homeostasis of water body. The toxicity of AgNPs to microalgae are known to be related to 
photosynthetic efficiency inhibition, reactive oxygen species (ROS) generation, metabolism interference, and organelles damage (He et al. 2017; Dorobantu et al. 2015). Whereas, the research concerning the toxic effects of AgNPs with multi-morphologies on algae were still limited. The investigation of structureactivity relationship of between AgNPs and algae is of great scientific value to the comprehensive and indepth understanding of AgNPs biological toxicity in aquatic environment and the evaluation of safety of water body.

Herein, we compared the toxicities of three AgNPs of various morphologies (AgNSs, AgNCs, and AgPLs) on an alga, Chlorella vulgaris. We determined the growth condition, the chlorophyll-a content, antioxidant enzyme activity, lipid peroxidation degree, and cell apoptosis. The experimental results of study could provide valuable information about the toxicity of AgNPs with various microstructure morphologies to aquatic organisms, which might be useful for assessing ecological risk of AgNPs.

\section{Materials And Methods \\ 2.1 Tested algae}

Experiments were carried out with cultures of the unicellular green algae $C$. vulgaris FACHB-8, purchased from Freshwater Algae Culture Collection at the Institute of Hydrobiology (Wuhan, China).

\subsection{Algae growth inhibition test}

Pre-cultures of $C$. vulgaris at exponential phase were inoculated into BG11 medium (Table S1) with an initial cell density of $10 \times 10^{6} \mathrm{cells} / \mathrm{ml}$ for algae growth and chlorophyll-a content assay. Before the inoculation of algae cells, different concentrations of AgNPs with various morphologies were added into the growth media. The nominal concentrations for AgNPs for the test in the medium were $0.5 \mathrm{mg} \mathrm{L}^{-1}, 1.0$ $\mathrm{mg} \otimes \mathrm{L}^{-1}, 2.0 \mathrm{mg} \otimes \mathrm{L}^{-1}, 5.0 \mathrm{mg} \varangle \mathrm{L}^{-1}$ (AgNPs concentrations verified by inductively coupled plasma mass spectrometry (ICP-MS) showed relatively small deviation). In the exposure test, cultures were grown in $150 \mathrm{ml}$ Erlenmeyer flasks containing $30 \mathrm{ml}$ of BG11 medium containing different concentrations of AgNPs.

Algae cells were grown in medium containing various AgNPs morphologies and concentration for $72 \mathrm{~h}$ and algae growth was monitored after every $12 \mathrm{~h}$. Cell density was measured by cell counting using an optical microscope (Nikon, China). The method proposed by Sartory (1984) was used to determine the content of photosynthetic pigment.

\subsection{Analysis of antioxidant enzyme activity and lipid peroxidation}

Cells at $48 \mathrm{~h}$ were harvested and analyzed for antioxidant enzyme activity and lipid peroxidation analysis. Superoxide dismutase (SOD) activity was assayed by monitoring the inhibition of reduction of Nitroblue Tetrazolium chloride (NBT) photochemically. The determination of Malondialdehyde (MDA) content was 
accomplished by the color reaction of Thiobarbituric Acid (TBA) in acid condition (Heath et al. 1968). The ROS level of the algae cells were measured using the ROS assay kit (Beyotime Institute of Biotechnology, Haimen, China). The activity of Peroxidase (POD) and Catalase (CAT) were determined by guaiacol method and UV absorption method respectively.

\subsection{Analysis of cells apoptosis}

After exposure of $48 \mathrm{~h}$, centrifuged at $4000 \mathrm{rmp}$ for $10 \mathrm{~min}$ at room temperature and cultured with $195 \mu \mathrm{L}$ Annexin V-FITC binding solution and $5 \mu \mathrm{L}$ Annexin V-FITC for $10 \mathrm{~min}$ at $24^{\circ} \mathrm{C}$. Next, cells were assayed using an Annexin V-FITC apoptosis detection kit (Beyotime Institute of Biotechnology, Haimen, China), the cell apoptosis and cell size were analyzed on a FACS JAZZ flow cytometer (Becton Dickinson, Sanjose, CA, USA) equipped with an argon laser (excitation at $488 \mathrm{~nm}$ ).

\section{Result And Discussion}

\subsection{Growth inhibition test}

During the experimental process, AgNPs with different concentrations $\left(0.5,1,2,5 \mathrm{mg} \otimes \mathrm{L}^{-1}\right)$ had different morphology response to the tested algae. Compared to the control, $C$. vulgaris was highly sensitive to AgPLs than the others, which showed significantly disruption and aggregation (Fig. 1A). After $48 \mathrm{~h}$ exposure in AgNPs, evident whitening and stunting appeared on algae cells. The result shows that the presence of AgNPs could lead to the aggregation and even rupture of algae cells, thus affecting their normal physiological and biochemical functions. The $48 \mathrm{~h}$ inhibition rate of $C$. vulgaris exposed to AgNSs, AgNCs and AgPLs increased with the concentration increasing in a dose related manner (Fig. 1B) $(\mathrm{P}<$ 0.5). However, tested concentrations of AgNSs and AgNCs did not induce substantial inhibition on cell growth, with the maximum inhibition effect of $43 \%$ and $38 \%$ at $5 \mathrm{mg} ه \mathrm{~L}^{-1}$. On the contrary, low concentration of AgPLs $\left(0.5 \mathrm{mg} \otimes \mathrm{L}^{-1}\right)$ could repress cell growth significantly by $60 \%$, indicating a higher toxic effect on cell growth and vitality than AgNSs and AgNCs in this study.

The inhibitory effects of AgNSs, AgNCs and AgPLs on the growth and photosynthetic of $C$. vulgaris are shown in Fig. $1 \mathrm{C}-\mathrm{H}$. The cell density of microalgae is the most intuitive parameter to measure the cell growth, and the chlorophyll-a content can be used as one of the indicators to measure the physiological effects of algae cells (Metzler et al. 2012). It can be seen from the figure that the presence of AgNPs inhibited the cells growth and chlorophyll-a synthesis, the inhibition efficiency had a positive relationship with the concentration of AgNPs. Incubation of algae cells in medium containing AgPLs resulted in significant repression on cell growth even at lower concentration of $0.5 \mathrm{mg} \otimes \mathrm{L}^{-1}$ at $72 \mathrm{~h}$. Increase of the AgNPs concentration to $5 \mathrm{mg} \rrbracket^{-1}$ impeded $C$. vulgaris propagation completely. A similar decrease in chlorophyll-a was observed in AgNPs treatment at 0.5, 1, 2 and $5 \mathrm{mg} \mathrm{L}^{-1}$, which was in agreement with the cell density histogram. The content of chlorophyll-a was only $26.92 \%$, compared with the control group, as the concentration of AgPLs was $5 \mathrm{mg} \mathrm{LL}^{-1}$. The chlorophyll-a content and cell density of AgNSs decreased the least, that is, the effect of AgNSs on photosynthetic pigments activity and growth of algae 
was the weakest. Substantial decreased in chlorophyll-a contents of algae exposed to AgNPs indicated that the photochemical activity was repressed and occurrence of photoinhibition was inevitable. For AgNPs with different morphologies, flake nano silver has a stronger biocidal effect than spherical nanoparticles ( $\mathrm{Pal}$ et al. 2007), the experimental results confirm this viewpoint to some extent.

\subsection{Physiological and biochemical test}

ROS and MDA are direct indicators of lipid peroxidation and oxidative damage in algae system (Gao et al. 2020). Figure 2 shows the intracellular ROS level (Fig. 2A) and MDA contents (Fig. 2B) of C. vulgaris as a function of the AgNPs concentration. The intracellular ROS level was significantly promoted by increasing AgNPs concentration, whereas relative MDA activity gradually decreased with increasing AgNPs concentrations, resulting a minimum value at 5 of AgNPs. After exposure to $5 \mathrm{mg} \mathrm{L}^{-1} \mathrm{AgPLs}$ for $48 \mathrm{~h}$, level of the ROS was the highest, and was 2.7-times those of the control, accordingly. As shown in Fig. 2B, the content of MDA was higher than that of the control group, while MDA content exposed to AgPLs increased with the increase of concentration. MDA is one of the products of lipid peroxidation (Gaschler et al. 2017), the content of MDA will be lower than that of the control group when the cells were damaged and ruptured. The results showed that $C$. vulgaris was more sensitive to the AgPLs in terms of MDA and ROS.

In order to determine the effects of AgNPs with different morphologies on enantioselective oxidative stress of $C$. vulgaris, AgNSs, AgNCs and AgPLs exposure group were examined. As seen in Fig. 3, the SOD (Fig. 3A), CAT (Fig. 3B) and POD (Fig. 3C) exhibited similar trend upon exposure to AgNPs after 48h exposure. AgNPs with different morphologies tested differently affected the antioxidant enzyme activities in the algal cells. When $C$. vulgaris cells exposed to different concentrations of $\operatorname{AgNPs}(0.5,1,2$ and $5 \mathrm{mg} \rrbracket$ $\left.\mathrm{L}^{-1}\right)$, SOD activities were enhanced compared with those untreated cells. Besides, the increase of SOD and CAT were most obvious in the AgPLs treated group. SOD enzyme can decompose $\mathrm{O}_{2}{ }^{-}$, CAT and POD enzymes are involved in the decomposition of hydrogen peroxide, thus eliminating the influence of ROS. As expected, under the stimulation of AgNPs, the activity of antioxidant enzymes and the antioxidant capacity increased respectively. In this study, the enhancement on CAT, SOD and POD of cultures exposed to AgNPs signify the involvement of antioxidant enzyme in the antioxidant defense against the ROS. Oxidative stress could enhance the antioxidant capacity of cells, and they couldn't maintain internal stability when the content of reactive oxygen species increased to a certain amount.

\subsection{Effect of AgNPs on cells membrane damage}

The effects of AgNPs on apoptosis of $C$. vulgaris were evaluated by Annexin V-FITC/PI staining. Cell membrane damage was the primary manifestation of apoptosis (Kundrát et al. 2016), when the cell membrane damaged, Annexin $\mathrm{V}$ could bind to phosphatidylserine on the surface of cell membrane, and the fluorescence intensity could be detected by FITC and PI dyes. In flow cytometry image, upper left and upper right, lower right quadrants show percentage of early apoptotic cells, advanced stage of apoptotic cells and dead cells, lower left quadrant shows the percentage of live cells respectively. As shown in Fig. 4A, B and C, after exposure to AgNPs with various morphologies for 48 hours, the cell size, 
complexity and apoptosis of the experimental group were higher than those of the control group. The percentage of apoptotic cells increased to $80 \%$ under the AgPLs treatment, while that in AgNSs treated group was only about $50 \%$, and in AgNCs treated group was about $70 \%$. The result shows that $5 \mathrm{mg} \mathbb{\mathrm { L }}-1$ AgNPs could promote the apoptosis of $C$. vulgaris cells, and the degree of promotion was related to the morphology of AgNPs, AgPLs had the greatest damage to the cell membrane compared with AgNSs and AgNCs. Although AgNSs had the smallest nanoscale, it showed the lowest toxicity to $C$. vulgaris, this experimental phenomenon was markedly different from the published literature (Nam et al. 2019).

\section{Conclusion}

In this study, the toxicity differences of AgNSs, AgNCs and AgPLs in C. vulgaris were compared based on the growth inhibition, photosynthetic pigment content, antioxidant enzyme activity, lipid peroxidation, morphology and apoptosis of the cells. The growth and photosynthetic pigment content of $C$. vulgaris were significantly inhibited by all three AgNPs. The results of antioxidant enzymes activities and cell apoptosis indicated that AgPLs possessed the highest potent toxicity to $C$. vulgaris, followed by AgNCs (moderate) and AgNSs (lowest). The experimental findings indicated that the toxic effects of AgNPs on aquatic organisms may primarily depend on its microstructure morphology rather than on its nano size in some certain cases, especially upon exposure to $C$. vulgaris. This work pointed out that the microstructure morphology effects and nanoscale effects are of equal importance for understanding the toxicities of AgNPs in aquatic primary producers, whereas the former may results in more potential impact than the latter when it is exposed to some certain algae, e.g., C. vulgaris.

\section{Declarations}

\section{Acknowledgement}

This work was supported by the Fundamental Research Funds for the Central Universities (grant numbers N2001016 and N2001012), the Key Technologies Research and Development Program (grant number 2019YFC1803804) and National College Students Innovation and Entrepreneurship Training Program (grant number 210047).

\section{Authors contributions}

Conceived and designed the experiments: NCD, HBL and YHL. Performed the experiments: NCD, HBL and YHL. Analyzed the data and prepared the figures: NCD, HBL, YHL, FM, MSW, ZL and XC. Wrote the paper: NCD, HBL and YHL. Reviewed and commented on the paper: NCD, HBL, JNX, RC and HXW. All authors read and approved the manuscript $\square$

Data availability Not applicable.

Compliance with ethical standards. 
Conflict of interest The authors declare that they have no conflict of interest.

Ethical approval Not applicable.

Consent to participate Not applicable.

Consent to publish Not applicable.

\section{References}

1. Babak S, Farshid SG, Hashemi M, Nezhad HR, Nasrollahi A, Sima A, Sahar A (2012) Comparison of the anti-bacterial activity on the nanosilver shapes: nanoparticles, nanorods and nanoplates. Adv Powder Technol 23(1):22-26. https://doi.org/10.1016/j.apt.2010.11.011

2. Dale AL, Casman EA, Lowry GV, Lead JR, Viparelli E, Baalousha M (2015) Modeling nanomaterial environmental fate in aquatic systems. Environ Sci Technol 49(5):2587-2593. https://doi.org/10.1021/es505076w

3. Dorobantu LS, Fallone C, Noble AJ, Veinot J, Burrell RE (2015) Toxicity of silver nanoparticles against bacteria, yeast, and algae. J Nanopart Res 17(4):1-13. https://doi.org/10.1007/s11051-015-2984-7

4. Gao J, Wang F, Jiang W, Han J, Zhou Z (2020) Biodegradation of Chiral Flufiprole in Chlorella pyrenoidosa: Kinetics, Transformation Products, and Toxicity Evaluation. Journal of agricultural and food chemistry. 68(7):1966-1973. https://doi.org/10.1021/acs.jafc.9b05860.

5. Gaschler MM, Stockwell BR (2017) Lipid peroxidation in cell death. Biochem Biophys Res Commun 482(3):419-425. https://doi.org/10.1016/j.bbrc.2016.10.086

6. He M, Yan Y, Pei F, Wu M, Gebreluel T, Zou S, Wang C (2017) Improvement on lipid production by Scenedesmus obliquus triggered by low dose exposure to nanoparticles. Sci Rep 7(1):1-12. https://doi.org/10.1038/s41598-017-15667-0

7. Heath RL, Packer L (1968) Photoperoxidation in isolated chloroplasts: I. Kinetics and stoichiometry of fatty acid peroxidation. Arch Biochem Biophys 125(1):189-198. https://doi.org/10.1016/00039861(68)90654-1

8. Ji E, Kim S, Jin H, Youn P, Ryu D (2010) Induction of oxidative stress and apoptosis by silver nanoparticles in the liver of adult zebrafish. Aquat Toxicol 100(2):151-159.

https://doi.org/10.1016/j.aquatox.2009.12.012

9. Kundrát $P$, Friedland W (2016) Enhanced release of primary signals may render intercellular signalling ineffective due to spatial aspects. Sci Rep 6(1):1-12. https://doi.org/10.1038/srep33214

10. Mayer C., Epple M., Sengstock Gross-Heitfeld., Koeller『Schildhauer A. T.『Helmlinger., 2016. Silver nanoparticles with different size and shape: equal cytotoxicity, but different antibacterial effects. RSC Adv 6(22):18490-18501. https://doi.org/10.1039/c5ra27836h

11. Metzler DM, Erdem A, Tseng YH, Huang C (2012) Responses of algal cells to engineered nanoparticles measured as algal cell population, chlorophyll a, and lipid peroxidation: effect of 
particle size and type. J Nanotechnol 2(2012):87-98. https://doi.org/10.1155/2012/237284

12. Mo F, Li H, Li Y, Cui W, Wang H (2020) Toxicity of ag+ on microstructure, biochemical activities and genic material of trifolium pratense $\mathrm{l}$. seedlings with special reference to phytoremediation. Ecotoxicol Environ Saf 195:110499. https://doi.org/10.1016/j.ecoenv.2020.110499

13. Nam SH, An YJ (2019). Size- and shape-dependent toxicity of silver nanomaterials in green alga Chlorococcum infusionum. Ecotoxicol Environ Saf 168:388-393. https:// doi.org/ 10.1016/j.ecoenv.2018.10.082.

14. Oukarroum A, Bras S, Perreault F, Popovic R (2012) Inhibitory effects of silver nanoparticles in two green algae, chlorella vulgaris and dunaliella tertiolecta. Ecotoxicol Environ Saf 78:80-85. https://doi.org/ 10.1016/j.ecoenv.2011.11.012

15. Pal S, Yu KT, Song JM (2007) Does the antibacterial activity of silver nanoparticles depend on the shape of the nanoparticle? A study of the gram-negative bacterium Escherichia coli. Appl Environ Microbiol 73(6):1712-1720. https://doi.org/10.1128/AEM.02218-06

16. Sartory DP, Grobbelaar JU (1984) Extraction of chlorophyll a from freshwater phytoplankton for spectrophotometric analysis. Hydrobiologia 114(3):177-187. https://doi.org/10.1007/BF00031869

17. Shen Y, Wang W, Fan A, Wei D, Liu W, Han C, Shen Y $₫$ Meng D, San X (2015) Highly sensitive hydrogen sensors based on $\mathrm{SnO} 2$ nanomaterials with different morphologies. Int J Hydrog Energy 40(45):15773-15779. https://doi.org/10.1016/j.ijhydene.2015.09.077

18. Wang M, Li H, Li Y, Mo F, Wang H (2020) Dispersibility and size control of silver nanoparticles with anti-algal potential based on coupling effects of polyvinylpyrrolidone and sodium tripolyphosphate. Nanomaterials 10(6):1042. https://doi.org/10.3390/nano10061042

\section{Figures}



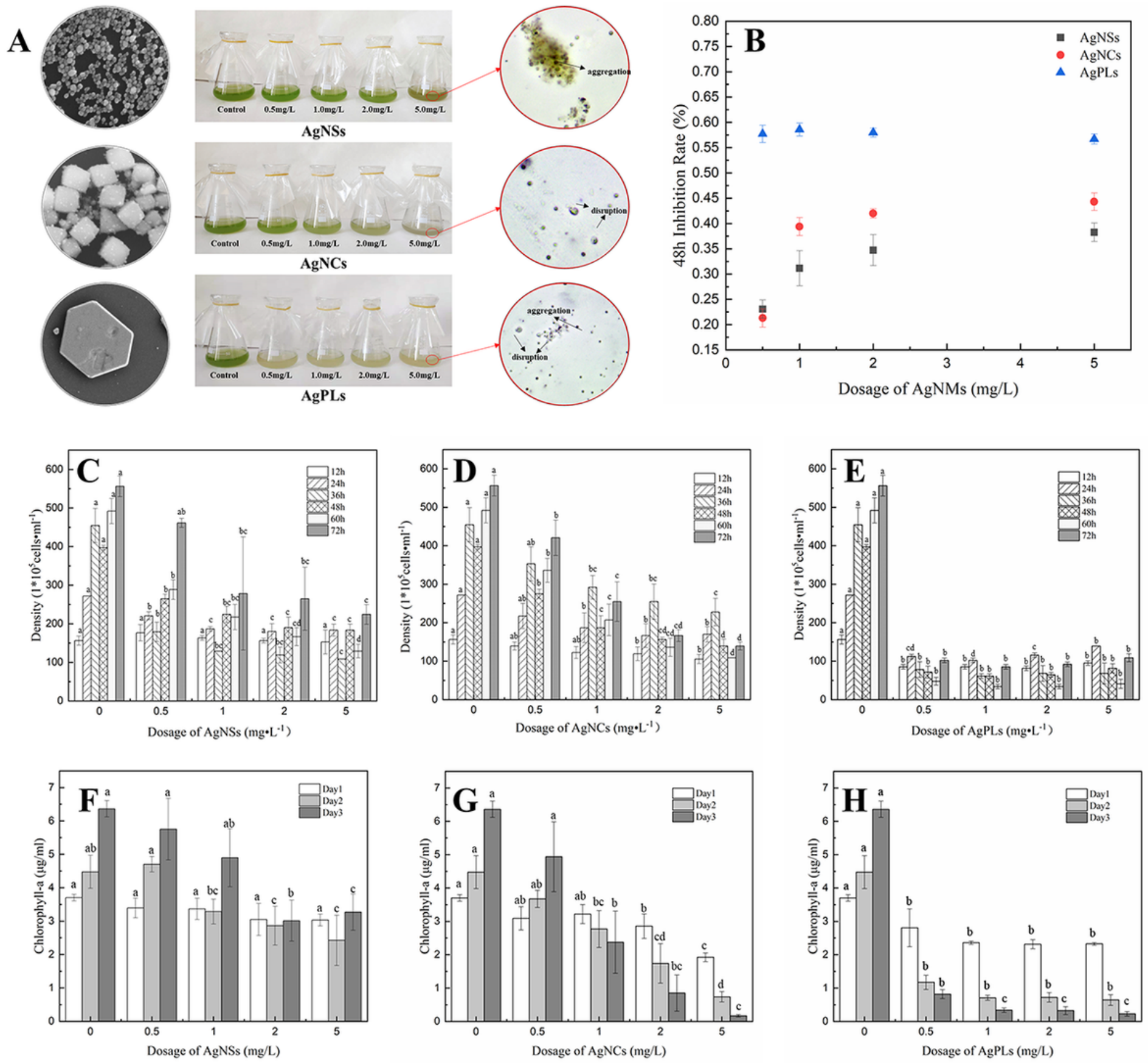

\section{Figure 1}

Determination of AgNPs SEM and cell morphology observation, 48h inhibition rates, cell density and chlorophyll-a contents of $\mathrm{C}$. vulgaris exposed to AgNPs with various morphologies for 3 days. AgNPs SEM images and cell morphology (A). 48h inhibition rates (B). Cell density (C, D, E). Chlorophyll-a content $(F, G, H)$. Lower-case letters demonstrate averages comparisons between treatments by LSD test $(p<$ $0.05)$. 

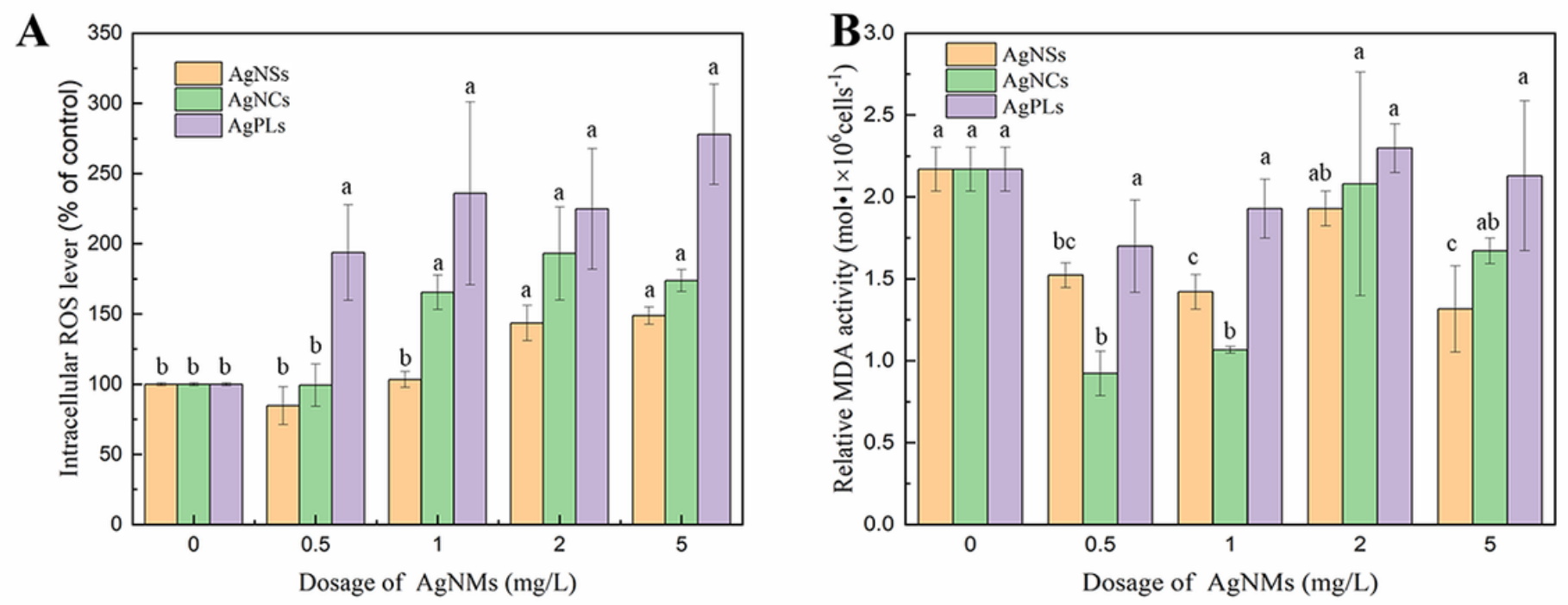

Figure 2

Changes of intracellular ROS level (A), MDA content (B) in C. vulgaris exposed to AgNPs with different concentrations after $48 \mathrm{~h}$. Lower-case letters demonstrate averages comparisons between treatments by LSD test $(p<0.05)$.

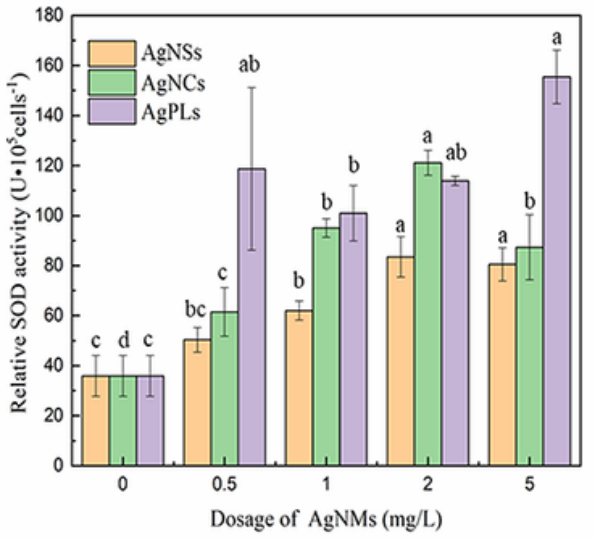

A

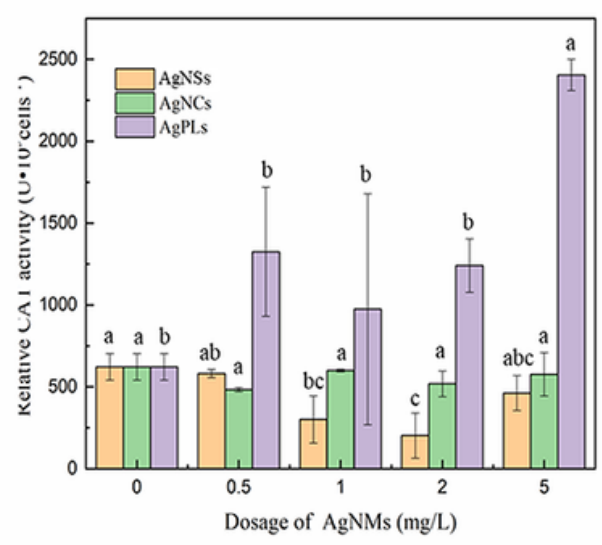

B

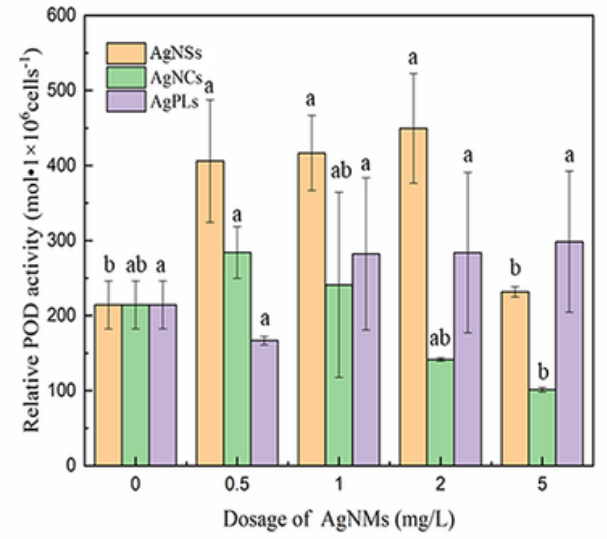

C

\section{Figure 3}

Changes of SOD (A), CAT (B) and POD (C) in C. vulgaris at different concentrations exposed to AgNPs with different concentrations after $48 \mathrm{~h}$. Lower-case letters demonstrate averages comparisons between treatments by LSD test $(p<0.05)$. 


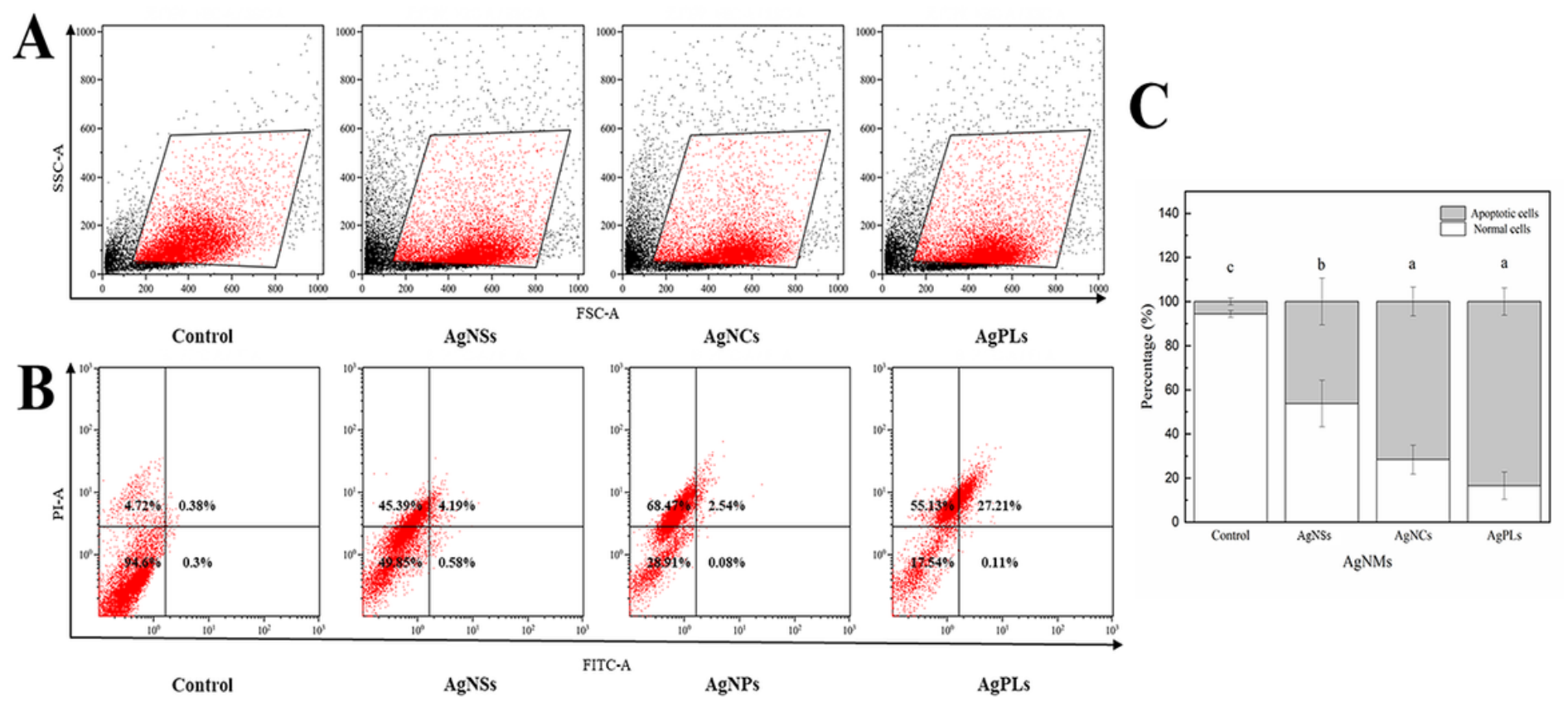

Figure 4

The effect of AgNPs on cell apoptosis in C. vulgaris. The cells were treated with different morphologies of AgNPs in the concentration of $5 \mathrm{mg} \varangle \mathrm{L}-1$ for $48 \mathrm{~h}$, then cells were collected for Annexin V-FITC/PI staining followed by cytometry analysis. (A) Morphology and size distribution of cells in different AgNPs treatment groups. (B) Apoptosis of cells in different AgNPs treatment groups. (C) Percentage of apoptotic and normal cells. Lower-case letters demonstrate averages comparisons between treatments by LSD test $(p<0.05)$.

\section{Supplementary Files}

This is a list of supplementary files associated with this preprint. Click to download.

- SupplementaryInformation.pdf

- GraphicalAbstact.png 